\title{
Correction to: New Psychoactive Substances (NPS), Psychedelic Experiences and Dissociation: Clinical and Clinical Pharmacological Issues
}

\author{
Fabrizio Schifano ${ }^{1,2}$. Flavia Napoletano ${ }^{3}$ Stefania Chiappini ${ }^{1,4}$ - Laura Orsolini ${ }^{1}$ - Amira Guirguis ${ }^{5}$. \\ John Martin Corkery ${ }^{1} \cdot$ Stefania Bonaccorso ${ }^{2} \cdot$ Angelo Ricciardi $^{2,6} \cdot$ Norbert Scherbaum $^{7}$ - Alessandro Vento ${ }^{8,9,10}$
}

Published online: 7 May 2019

(C) Springer Nature Switzerland AG 2019

\section{Correction to: Current Addiction Reports https://doi.org/10.1007/s40429-019-00249-z}

The original version of this article unfortunately contained a mistake. Authors' first names and family names were interchanged.

The original version has been corrected.

Publisher's Note Springer Nature remains neutral with regard to jurisdictional claims in published maps and institutional affiliations.

The online version of the original article can be found at https://doi.org/ 10.1007/s40429-019-00249-Z

Stefania Chiappini

stefaniachiappini9@gmail.com

Fabrizio Schifano

f.schifano@herts.ac.uk

Flavia Napoletano

flavia.napoletano@gmail.com

Laura Orsolini

laura.orsolini01@gmail.com

Amira Guirguis

amira.guirguis@swansea.ac.uk
John Martin Corkery

j.corkery@hert.ac.uk

Stefania Bonaccorso

stefania.bonaccorso@kcl.ac.uk

Angelo Ricciardi

ricciardi.angelo@gmail.com

Norbert Scherbaum

norbert.scherbaum@uni-due.de

Alessandro Vento

alessandrovento@gmail.com

Extended author information available on the last page of the article 


\section{Affiliations}

Fabrizio Schifano ${ }^{1,2}$. Flavia Napoletano ${ }^{3}$ - Stefania Chiappini ${ }^{1,4} \cdot$ Laura Orsolini $^{1}$ • Amira Guirguis ${ }^{5}$. John Martin Corkery ${ }^{1} \cdot$ Stefania Bonaccorso $^{2} \cdot$ Angelo Ricciardi $^{2,6} \cdot$ Norbert Scherbaum $^{7} \cdot$ Alessandro Vento $^{8,9,10}$

1 Psychopharmacology, Drug Misuse and Novel Psychoactive Substances Research Unit, School of Life and Medical Sciences, University of Hertfordshire, College Lane Campus, Hatfield AL10 $9 \mathrm{AB}, \mathrm{UK}$

2 Camden and Islington NHS Mental Health Foundation Trust, 4 St Pancras Way, London NW1 0PE, UK

3 East London Foundation Trust (ELFT), Homerton University Hospital, 9 Alie St, E1 8DE London, UK

4 Casa di Cura Villa Rosa, Via Francesco Baracca 21, 01100 Viterbo, Italy
5 Swansea University Medical School, Institute of Life Sciences 2, Swansea University, Singleton Park, SA 28PP, Swanseaw, Wales

6 Department of Mental Health, ASL Roma 1, Rome, Italy

7 LVR-Klinikum Essen, Klinik fur Psychiatrie und Psychotherapie, University of Duisburg-Essen, Wickenburgstraße 21, 45147 Essen, Germany

8 Addictions' Observatory (ODDPSS), Rome, Italy

9 Guglielmo Marconi University, Via Plinio, 44, 00193 Rome, Italy

10 Department of Mental Health, ASL Roma 2, Rome, Italy 